\title{
The therapeutic effects of traditional chinese medicine on COVID-19: a narrative review
}

\author{
Can Wang ${ }^{1} \cdot$ Shusen Sun ${ }^{2,3,4} \oplus \cdot$ Xuansheng Ding $^{1}$
}

Received: 8 May 2020 / Accepted: 11 September 2020 / Published online: 24 September 2020

(c) Springer Nature Switzerland AG 2020

\begin{abstract}
Background The Coronavirus Disease 2019 (COVID-19) is a new, highly infective virus. In the absence of specific vaccines and antiviral drugs, the China National Health Commission has released a series of COVID-19 treatment guidelines, which include symptomatic treatment, antiviral treatment, and traditional Chinese medicines (TCM). Aim of the review To analyze and summarize the role of TCMs in the treatment of COVID-19. Methods Relevant studies on TCMs related to the study aim were undertaken through a literature search to synthesize the extracted data. Results The China National Health Commission guidelines recommend 15 oral and injectable TCMs for COVID-19. Studies on TCMs have demonstrated that these compounds possess broad-spectrum antiviral and antibacterial properties, and they have certain advantages in the treatment of viral pneumonia and other emergent infectious diseases. Many TCMs also have potent anti-inflammatory and immunomodulatory effects. Treatment based on syndrome differentiation is one of the significant features in TCM. TCM compounds contain a variety of active ingredients that have proven multi-target effects, making it difficult for viruses to develop drug resistance. A variety of clinical pathways are involved in the actions of these TCMs. These properties allow TCMs to be used in the treatment of COVID-19. Conclusion TCMs have been widely used in the treatment of COVID-19 in China. The unique properties of TCM compounds allow TCMs to have a role in the management of COVID-19.
\end{abstract}

Keywords Chinese medicine $\cdot$ Coronavirus · COVID-19 · Diagnosis $\cdot$ Traditional chinese medicine (TCM) · Treatment

\section{Impacts on practice}

- Compounds of Traditional Chinese medicines (TCM) often possess several therapeutic properties due to multiple compositions and signaling pathways.

Shusen Sun

ssun@wne.edu

$\bowtie$ Xuansheng Ding dxs0162@sina.com

1 Department of Pharmacy, School of Basic Medicine and Clinical Pharmacy, China Pharmaceutical University, Nanjing 211198, China

2 Department of Pharmacy Practice, College of Pharmacy and Health Sciences, Western New England University, Springfield, MA 01119, USA

3 Department of Pharmacy, Xiangya Hospital, Central South University, Changsha 410008, Hunan, China

4 The Hunan Institute of Pharmacy Practice and Clinical Research, Changsha 410008, Hunan, China
- Treatment of COVID-19 using TCMs can be based on syndrome differentiation, a unique feature of traditional Chinese medicine.

- Traditional Chinese medicines can be considered for the treatment of COVID-19 based on TCM theories.

\section{Introduction}

Novel coronavirus pneumonia (COVID-19) is highly infectious and has caused widespread infection [1,2]. To guide the diagnosis and treatment of COVID-19, the China National Health Commission (NHC) has successively released and updated "The COVID-19 Diagnosis and Treatment Plan (NHC guidelines)" [3-6]. The NHC guidelines include the route of transmission, pathological features, clinical manifestations, etiology, serological examination, diagnostic criteria, clinical typing, treatment regimens, and discharge criteria. Particular emphasis is placed on the clinical condition of severe and critical patients, rational development of personalized, integrated traditional Chinese 
medicine (TCM), and western medicine diagnosis and treatment. Appropriate therapy should be selected according to the severity and clinical manifestations [6].

TCM has a long history in treating infectious diseases in China. TCM can rapidly improve symptoms and reduce the occurrence of complications through syndrome differentiation. Syndrome differentiation is a summary of the pathological signs of the body at a specific stage during disease development, based on the data collected by the synthesis of the four diagnostic methods: observation, listening and smelling, asking, and palpation and pulse-taking. Thus, TCM is recommended as a treatment option of COVID-19 in the NHC guidelines [6]. However, the efficacy of TCM on COVID-19 remains to be verified. We aimed to fill in the gap by evaluating the current literature and provide help for clinical decision-making.

\section{Aim of the review}

The objective of this review was to analyze and summarize the clinical manifestations, etiology, and pathogenesis of COVID-19 and the rational application of Chinese medicine from the perspective of TCM.

\section{Methods}

Studies were identified through searching in PubMed, Embase, the Cochrane Library, China National Knowledge Infrastructure (CNKI), and the China Wanfang database. Both CNKI and China Wanfang are the two most authoritative, comprehensive, and the most abundant sources in China. Studies and reviews were limited to those in English or Chinese concerning the use of TCMs in COVID19. Additional articles were identified through cross-referencing articles published in either English or Chinese. We also searched websites of the China NHC, and the China Food and Drug Administration (CFDA) to identify relevant information. The following grouped keywords were used as search strategy and modified according to different databases: ("coronavirus disease 2019" OR "COVID-19" OR "SARS-CoV-2" OR "novel coronavirus pneumonia" OR "novel coronavirus") AND ("Chinese herbal medicine" OR "traditional Chinese medicine" OR "classical Chinese herbal formulas" OR "Chinese herb" OR "Chinese herb therapy" OR "herbal medicine" OR "herb therapy" OR "herbal remedy") AND ("clinical trial" OR "clinical study" OR "randomized controlled trial").

Our research team includes members practicing in the US and China and are fluent in English and Chinese. Relevant information was independently searched and extracted, and disagreement was settled through discussion to maintain the validity and reliability. The translation of TCM terms refers to the "WHO International Standard Terminologies on Traditional Medicine in the Western Pacific Region" [7], and the "International Standard Chinese-English Basic Nomenclature of Chinese Medicine" [8].

\section{Results}

\section{The etiology, pathogenesis, diagnosis, and treatment of COVID-19 from TCM perspectives}

COVID-19 is considered a febrile disease and epidemic in TCM. The patients' clinical symptoms and the corresponding treatment have been summarized in the NHC guidelines. According to disease development, the clinical stages of COVID-19 are divided into the observation stage and clinical treatment stage (light, medium, severe, critical, and recovery stage), with different clinical manifestations (Table 1). COVID-19 belongs to the category of epidemic gas based on TCM, which is very contagious. The virus enters the body via the mouth and nose, then damages the lungs, spleen, and stomach as it grows and multiplies [9]. From a TCM perspective, COVID-19 has a rapid onset, rapid spread, strong infectivity, similar main symptoms, and seasonality [10].

In the NHC guidelines, a total of 15 TCMs are recommended, of which 7 are taken orally (Angong Niuhuang, Zixue, Huoxiang Zhengqi, Jinhua Qinggan, Lianhua Qingwen, Shufeng Jiedu, and Fangfeng Tongsheng), and 8 are injections (Xiyanping, Xuebijing, Reduning, Tanreqing, Xingnaojing, Shenfu, Shengmai, and Shenmai) (Table 2). The active constituents of these preparations are presented in Table 3.

\section{Application of oral TCMs in the treatment of COVID-19}

\section{Jinhua Qinggan granules}

Jinhua Qinggan granules are composed of honeysuckle, gypsum, ephedra (honey), bitter almond, baicalin, forsythia, fritillaria, burdock seed, artemisia annua, mint, and licorice [11]. Jinhua Qinggan has traditionally been used for the treatment of plague diseases. However, clinical studies have shown that it has therapeutic efficacy against viral infections. In 2011, a clinical trial with 403 patients was conducted to verify the effectiveness of Jinhua Qinggan and oseltamivir in treating H1N1 influenza [11]. This study showed that when compared to placebo, the combination of Jinhua Qinggan and oseltamivir shortened the time patients experienced a fever $(P<0.001)$, with the fever time for patients in the combination group being 
Table 1 Clinical manifestations of TCM in different stages of COVID-19

\begin{tabular}{|c|c|c|}
\hline Stage & Clinical manifestation & \\
\hline \multirow[t]{2}{*}{ Medical observation period } & Clinical manifestation 1 & Hypodynamia and gastrointestinal discomfort \\
\hline & Clinical manifestation 2 & Hypodynamia and fever \\
\hline \multirow[t]{9}{*}{ Treatment period } & Light type & $\begin{array}{l}\text { Cold-damp encumbering the lung: fever, hypodynamia, body aches, cough, expectora- } \\
\text { tion, tight chest and breathless, anorexia, nausea, vomiting, loose stool, tongue bearing } \\
\text { dental impressions or red, white fur, soggy pulse or slippery pulse }\end{array}$ \\
\hline & & $\begin{array}{l}\text { Damp-heat retention in lung: low fever, aversion to cold, hypodynamia, heavy cumber- } \\
\text { some head and body, sore muscle, dry cough with little sputum, pharyngalgia, dry } \\
\text { mouth, oppression in the chest, anhidrosis, anorexia, loose stool, red tongue, light- } \\
\text { colored tongue with white and slippery coating, soggy pulse or slippery pulse }\end{array}$ \\
\hline & Moderate type & $\begin{array}{l}\text { Pattern damp toxin brewing and binding: fever, cough, low phlegm or yellow phlegm, } \\
\text { suffocating, abdominal distension, constipation, red tongue, fat tongue, yellow fur or } \\
\text { dry, soggy pulse or slippery pulse }\end{array}$ \\
\hline & & $\begin{array}{l}\text { Pattern of cold-damp encumbering the lung: Low fever, hiding fever, dry cough, dry } \\
\text { cough, scant phlegm, hypodynamia, oppression in chest, nausea and vomiting, loose } \\
\text { stool, red tongue, light-colored tongue with white and slippery coating, soggy pulse }\end{array}$ \\
\hline & Severe & $\begin{array}{l}\text { Pattern of epidemic toxin internal block: fever and blush, cough, phlegm yellow and } \\
\text { sticky, blood in phlegm, shortness of breath, hypodynamia, dry mouth, bitter taste in } \\
\text { mouth, nausea, constipation, yellow urine, red tongue, tongue yellow and greasy, slip- } \\
\text { pery pulse }\end{array}$ \\
\hline & & $\begin{array}{l}\text { Pattern of qi and construction both ablaze: great heat and thirst, shortness of breath, } \\
\text { delirium, blurred vision, or hematemesis, apostaxis, or convulsion of the limbs, no } \\
\text { tongue coating, sunken pulse, thread pulse, floating pulse }\end{array}$ \\
\hline & Critical type & $\begin{array}{l}\text { Internal block and outward desertion: difficulty breathing, Shortness of breath or needing } \\
\text { mechanical ventilation, dizziness, irritability, sweating and feeling cold, dark purple } \\
\text { tongue, thick or dry tongue coating, floating pulse }\end{array}$ \\
\hline & Recovery stage & $\begin{array}{l}\text { Dual vacuity of the lung and spleen: shortness of breath, hypodynamia, poor appetite, } \\
\text { glomus and fullness, constipation, loose stool, fat tongue, pale tongue }\end{array}$ \\
\hline & & $\begin{array}{l}\text { Pattern of dual vacuity of lung: weakness, shortness of breath, dry mouth, thirst, palpita- } \\
\text { tions, sweaty, poor appetite, low fever, dry cough with less phlegm, dry tongue, thready } \\
\text { pulse or feeble pulse }\end{array}$ \\
\hline
\end{tabular}

$T C M$ traditional Chinese medicine

$19 \%$ shorter than the oseltamivir group $(P=0.05)$. The results show that the combination of Jinhua Qinggan and oseltamivir have good efficacy in treating H1N1 influenza. Another study assessed Jinhua Qinggan in treating influenza patients and its impact on the serum cytokine level [12]. The results show patients who took Jinhua Qinggan significantly reduced the serum cytokine levels and enhanced the body's immune function.

Jinhua Qinggan have been used as adjuvant therapy for COVID-19 in clinical practice. In this study, 123 COVID19 outpatients were randomly divided into a treatment group (Jinhua Qinggan combined with routine treatment for 5 days, $n=82$ ) and a control group (routine treatment only, $n=41$ ). The addition of Jinhua Qinggan significantly alleviated fever, cough, fatigue, sputum, anxiety, and the hospitalization rate tended to be lower than in the control group [13]. Although a randomized controlled study, the sample size was small. Jinhua Qinggan is recommended for treating patients during the medical observation period in the NHC guidelines [6].

\section{Lianhua Qingwen capsules (granules)}

Lianhua Qingwen is a compound preparation of TCM based on the theory of prevention and treatment of plague, including forsythia, honeysuckle, ephedra, bitter almond, gypsum, isatis, mianma guanzhong, houttuynia cordata patchouli, rhubarb, rhodiola rosea, menthol, and licorice [14]. The active ingredients may be quercetin and luteolin oxalin, and kaempferol. These components are involved in broad-spectrum antiviral, antibacterial and antipyretic effects, cough and phlegm reduction, and immune regulation through targets such as mitogen-activated protein kinase and Hepatitis B signaling pathways [15]. It can inhibit the release of inflammatory mediators and reduce the lung tissue damage caused by inflammation [16]. The lung infection is related to lung immunity, and Lianhua Qingwen can regulate lung immunity [17].

Retrospective clinical analysis has indicated the role of Lianhua Qingwen in treating new coronavirus pneumonia [18]. One study enrolled 102 COVID-19 patients (1:1 


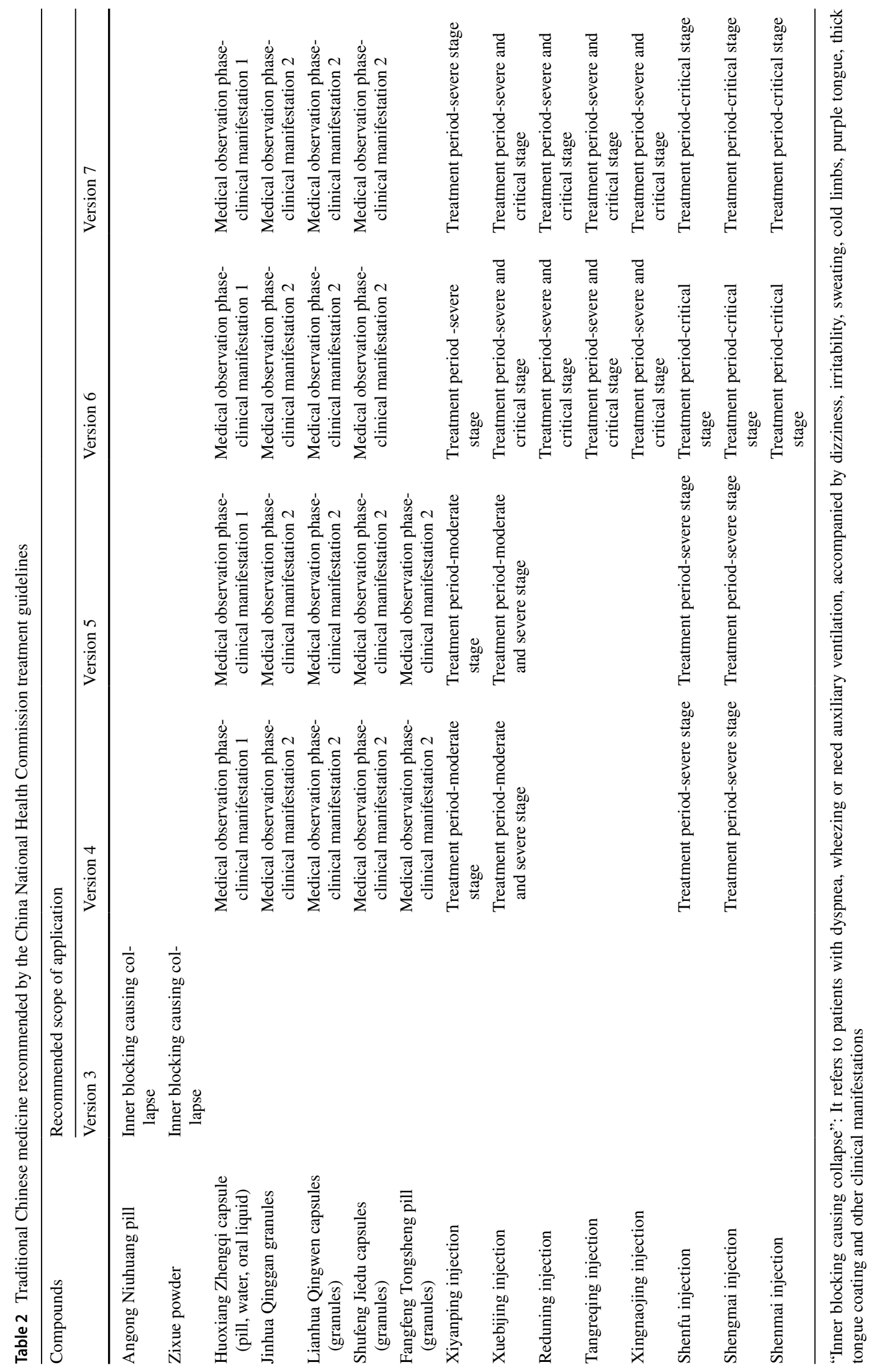


Table 3 The active constituents of traditional Chinese medicine recommended by the China National Health Commission treatment guidelines

\begin{tabular}{|c|c|}
\hline Compounds & Active constituents of the respective compounds \\
\hline Jinhua Qinggan granules & $\begin{array}{l}\text { Honeysuckle, gypsum, ephedra (honey), bitter almond, baicalin, forsythia, fritillaria, burdock } \\
\text { seed, artemisia annua, mint, licorice }\end{array}$ \\
\hline Lianhua Qingwen capsules (granules) & $\begin{array}{l}\text { Forsythia, honeysuckle, ephedra, bitter almond, gypsum, isatis, mianma Guanzhong, houttuynia } \\
\text { cordata patchouli, rhubarb, rhodiola rosea, menthol, licorice }\end{array}$ \\
\hline Shufeng Jiedu capsules (granules) & Polygonum cuspidatum, forsythia, radix isatidis, bupleurum, radix, verbena, reed root, and licorice \\
\hline Xiyanping injection & Andrographolide compound \\
\hline Xuebijing injection & Safflower, red peony, chuanxiong, salvia miltiorrhiza, and angelica \\
\hline Reduning injection & Honeysuckle, gardenia, and artemisia annua \\
\hline Tangreqing injection & Scutellaria baicalensis, bear bile powder, goat horn, honeysuckle, and forsythia \\
\hline Xingnaojing injection & Musk, turmeric, borneol, gardenia \\
\hline Shenfu injection & Red ginseng and black monkshood \\
\hline Shenmai injection & Red ginseng and ophiopogon japonicus \\
\hline Shengmai injection & Red ginseng, ophiopogon japonicus and schisandra chinensis \\
\hline
\end{tabular}

ratio), 51 were taking Lianhua Qingwen combined with conventional treatment in the treatment group, and 51 in the conventional treatment as the control group [19]. This trial compared the disappearance rate, duration, and effectiveness at relieving the main symptoms (fever, fatigue, and cough) between the two groups. The disappearance rate of other symptoms and signs and the improvement rate of lung computed-tomography (CT) were also analyzed. It was found that the combined application of Lianhua Qingwen, compared to conventional treatment, significantly improved the clinical symptoms of COVID-19 patients. The strength of the study was multicenter. However, it was a retrospective analysis. Another 14-day study randomized 284 COVID-19 patients into the control group (usual treatment only) or the treatment group (Lianhua Qingwen plus usual treatment) [20]. The rate of symptom recovery (fever, fatigue, coughing) was significantly higher in the treatment group compared with the control group $(91.5 \%$ vs. $82.4 \%, P=0.022)$. The rate of improvement in chest CT $(83.8 \%$ vs. $64.1 \%, P<0.001)$ and clinical cure $(78.9 \%$ vs. $66.2 \%, P=0.017$ ) were also higher in the treatment group. The study was a prospective multicenter randomized controlled trial. However, it was an open-label study. Lianhua Qingwen is currently recommended for treating patients during the clinical observation period and in the early stage of the disease (mild case) in the NHC guidelines [6].

\section{Shufeng Jiedu capsules (granules)}

Shufeng Jiedu capsules (granules) are mainly used for acute upper respiratory tract infections. The granules consist of polygonum cuspidatum, forsythia, radix isatidis, bupleurum, radix, verbena, reed root, and licorice [21]. It has been found that Shufeng Jiedu has a broad-spectrum antiviral effect. It has an inhibitory effect on influenza-A virus H1N1 type, herpes simplex virus type 1 and 2, respiratory syncytial virus, parainfluenza virus, Coxsackievirus B4, Coxsackievirus B5, and adenovirus [22]. The anti-inflammatory effects of Shufeng Jiedu are related to the inhibition of the MAPK/ NF-kB signaling pathway and the down-regulation of NF-kB mRNA expression [23]. Compounds such as iridoid glycosides and phenylethyl alcohol glycosides are the basis for inhibiting the expression of inflammatory factors [24]. This inhibitory effect is seen primarily through reducing the level of lactic acid, increasing the oxygen partial pressure in the lung tissue, promoting the absorption of lung inflammation, and relieving local symptoms. It plays an active role in the treatment of viral pneumonia. The biological approach of Shufeng Jiedu in the treatment of COVID-19 may be similar to the above-discussed properties. There are no reported clinical studies in the COVID-19 treatment. It is recommended for treating patients during the clinical observation and early stages of the disease (mild case) in the NHC guidelines [6].

\section{Application of TCM injections in the treatment of COVID-19}

\section{Xiyanping injection}

Xiyanping has the effects of clearing away heat and detoxification, curing cough, and dysentery. It is clinically used for the treatment of bronchitis, tonsillitis, and bacterial disease. Its main component is an andrographolide compound [25]. The diterpene bicyclic ring and the five-membered lactone ring in the structure of andrographolide are necessary for their anti-inflammatory and antipyretic activities. The position and number of double bonds determine their anti-inflammatory activity [26]. In 20 patients with acute bronchopneumonia treated 
with andrographolide injection, andrographolide can directly act on the medium that regulates body temperature to achieve the effect of restoring normal body temperature [27]. An in vitro experiment of andrographolide and its sulfonate against various influenza viruses showed that it could inhibit apoptosis, increase the survival rate of infected animals, reduce pathological lung changes, reduce viral load, and reduce inflammatory cytokine expression [28].

Xiyanping can reduce inflammation in COVID-19 patients and improve symptoms such as cough, fever, and rales in the lungs [29]. Discussed benefits include inhibiting viral replication and infection, inhibiting concurrent bacterial infections, improving the body's immunity, and improving liver function and cardiovascular damage. However, there are no reported clinical studies in the COVID-19 treatment. Xiyanping is recommended for treating patients in the progressive stage of COVID-19 (critical case) in the NHC guidelines [6].

\section{Xuebijing injection}

Xuebijing consists of safflower, red peony, chuanxiong, salvia miltiorrhiza, and angelica [30]. It is indicated to treat sepsis, systemic inflammatory response syndrome, and multiple organ dysfunction syndromes (MODS) [30]. Xuebijing is widely used in China for treating severe pneumonia, chronic obstructive pulmonary disease, acute respiratory distress syndrome, and other critical conditions/diseases. The combined application of Xuebijing based on conventional antiviral treatment can promote the absorption of lung lesions and improve efficacy [30]. It has been found that adding Xuebijing to patients with severe pneumonia based on conventional treatment can reduce the levels of IL- 6 and TNF- $\alpha$ in the plasma [31].

Using a retrospective method, researchers investigated 44 patients with light new coronavirus pneumonia [32]. Patients were divided into an observation group $(\mathrm{n}=22)$ and a control group $(n=22)$. The observation group received $50 \mathrm{~mL}$ Xuebijing intravenously and $100 \mathrm{~mL}$ $0.9 \%$ normal saline for 7 days. After treatment, the lung CT showed that the effective rate of lesion absorption and the overall effective rate in the observation group were higher than those in the control group $(P<0.05)$. It is believed that Xuebijing treatment in COVID-19 (light type) can safely promote the absorption of lung infection lesions and improve treatment efficacy. It is worth noting that this was only a retrospective small size analysis. Currently, Xuebijing is recommended for treating patients in the progressive stage of COVID-19 (critical case) in the NHC guidelines [6].

\section{Reduning injection}

Reduning is composed of honeysuckle, gardenia, and artemisia annua. The injection has been shown to have antipyretic, anti-inflammatory, and anti-viral pharmacological effects [33]. It has proven efficacy in the treatment of acute bronchitis and upper respiratory tract infections. Therefore, it has become a recommended anti-epidemic drug in the prevention and treatment of epidemics [6]. Reduning combined with biapenem was reported to have a better clinical effect in treating severe pneumonia, improving patients' clinical symptoms, improving blood gas indexes and lung function, and reducing serum inflammatory factor levels [34]. Research has found that quercetin, luteolin, rutin, and isorhamnetin in the Reduning injection have anti-inflammatory, antiviral, and immunomodulatory effects [35]. The mechanism of action may be through the IL-17, C-type lectin receptor, HIF-1, and other pathways acting on IL-6, CASP3, MAPK1, CCL2, and other targets. Reduning has a good therapeutic effect on lung injury and cardiovascular disease. However, there are no reported clinical studies in the COVID-19 treatment. Currently, Reduning is recommended for treating patients in the progressive stage of COVID-19 (critical case) in the NHC guidelines [6].

\section{Tanreqing injection}

Tanreqing consists of scutellaria baicalensis, bear bile powder, goat horn, honeysuckle, and forsythia [36]. It has effects of clearing heat and detoxification, as well as reducing cough and phlegm. It is mainly used in the clinical treatment of early pneumonia, acute bronchitis, acute exacerbation of chronic bronchitis, and lower respiratory tract infections [37]. Quercetin and luteolin in Tanreqing have anti-influenza A virus activity in vitro [38]. Studies show that quercetin can reduce the expression of TGF- $\beta 1, \alpha$-SMA, and TNF$\alpha$, inhibit the apoptosis of rat alveolar cells, and lessen the inflammation and fibrosis destruction of rat lung tissue [38]. Baicalin can reduce the inflammatory damage of lung tissue by inhibiting the expression of TNF- $\alpha$ and IL-1 $\beta$ [39]. Network pharmacology and molecular docking study speculate that Tanreqing can treat COVID-19 through the combined action of multiple components, targets, and pathways [40]. However, there are no reported clinical studies in the COVID-19 treatment. Currently, Tanreqing is recommended for treating patients in the progressive stage of COVID-19 (critical case) in the NHC guidelines [6].

\section{Xingnaojing injection}

Xingnaojing consists of musk, turmeric, borneol, gardenia, and other ingredients [41]. It is commonly used clinically for acute poisoning, viral encephalitis, craniocerebral injury, 
acute cerebrovascular disease, pulmonary encephalopathy, pneumonia, respiratory failure, and sepsis [42]. Studies have compared the antipyretic effects of Xingnaojing and Qingkailing on upper respiratory tract infection in adults [43]. The results showed that the total effective rate in the Xingnaojing treatment group was $91.4 \%$, which was better compared to the Qingkailing control group, with no apparent adverse reactions seen in the treatment group. Xingnaojing can shorten the progression of acute infectious fever, especially the infection caused by viruses. It can significantly reduce the time patients experience fever and effectively shorten the treatment time [44].

Xingnaojing combined with conventional treatment can effectively reduce the fever of patients with severe craniocerebral injury, increase the sober rate, and improve patients' quality of life [45]. Xingnaojing can effectively reduce the level of pro-inflammatory factors in pneumonia rats, improve the ability to resist oxidative stress, and regulate immunity [46]. Xingnaojing combined with azithromycin can reduce the levels of serum inflammation indexes of TNF- $\alpha$ and $\mathrm{CRP}$, increase the content of $\mathrm{CD} 3+$ and $\mathrm{CD} 4+$, and reduce the level of $\mathrm{CD} 8+$ when treating mycoplasmal pneumonia in the elderly. Also, they can improve the body's immune function and improve treatment effect by reducing the inflammation level [47]. Xingnaojing can inhibit the overexpression of serum CRP, IL- 6 , and TNF- $\alpha$ during the adjuvant treatment of ventilator-associated pneumonia. It can also reduce the possibility of an inflammatory response and multiple organ function damage [48]. No clinical studies of Xingnaojing in COVID-19. Given critical cases with COVID-19 might suffer from consciousness, it is recommended for treating patients in the progressive stage of COVID-19 (critical case) in the NHC guidelines [6].

\section{Shenfu injection}

Shenfu is made from red ginseng and black monkshood, commonly used in the treatment of cardiovascular and cerebrovascular diseases [49]. It can also be used alone or assist in the treatment of severe pneumonia, sepsis, multiple organ dysfunction, and tumors. Shenfu can effectively reduce the levels of pro-inflammatory cytokines TNF- $\alpha$, IL-6, IL-8, procalcitonin, and hypersensitive CRP in the serum of patients with sepsis, as well as improve the therapeutic effects [50]. Shenfu can reduce the level of IL-6, increase the number of peripheral blood CD3 +, CD4+, and CD8 +-T cells, maintain the pro-inflammatory/anti-inflammatory balance, thus improving the treatment efficacy in sepsis [51]. Also, Shenfu can significantly improve the cure rate of symptoms such as cough, sputum, and lung rales, increase the content of $\operatorname{IgG}$, IgM, and $\operatorname{IgA}$ in body fluids, significantly improving immune function [52]. When observing the treatment of Shenfu on elderly chronic lung infections, it was found that Shenfu can reduce the level of immunoglobulin ( $\operatorname{Ig} \mathrm{A}, \operatorname{IgG}, \operatorname{IgM}$ ) in patients' serum and reduce the value of serum procalcitonin. It can also improve the body's immunity through immune regulation while playing an active role in treating lung infections [53].

Shenfu combined with antibiotic treatment can shorten the time of fever, cough, and lung rales in patients with severe pneumonia, reduce the content of soluble E-selection (s E-selection) and von Willebrand factor (vWF), and improve the function of vascular endothelial cells, while reducing myocardial damage, and enhance blood gas and respiratory indicators [54]. Shenfu can significantly improve the mean arterial pressure, cardiac index, extravascular lung water index, and other hemodynamic indexes in patients with severe pneumonia [55]. It can additionally inhibit the HMGB1/NF- $\mathrm{KB}$ signaling pathway, thereby reducing the expression of pro-inflammatory cytokines TNF- $\alpha$ and IL- $1 \beta$, preventing the occurrence of cytokine storms [56, 57]. However, there are no reported clinical studies in the COVID-19 treatment. Currently, Shenfu is recommended for treating patients in the progressive stage of COVID-19 (critical case) in the NHC guidelines [6].

\section{Shenmai injection}

Shenmai is composed of red ginseng and ophiopogon japonicus [58]. It is used to treat coronary heart disease, viral myocarditis, chronic pulmonary heart disease, and neutropenia. It can improve the immune function of tumor patients. When used in combination with chemotherapy drugs, it has a synergistic effect and can reduce the side effects of chemotherapy drugs [59]. The main components of Shenmai injection are saponins, sugars, amino acids, flavonoids, lignans, organic acids, and other substances [60]. Shenmai can reduce the level of inflammatory factors, increase anti-inflammatory factors, and reduce the content of white blood cells, c-reactive protein, and procalcitonin when used for the adjuvant treatment of severe pneumonia. Shenmai improves the cure rate in severe pneumonia through its anti-inflammatory effects [61]. One study randomly divided 93 patients with ventilator-associated pneumonia into three groups. The three groups were given conventional treatment with high-dose and low-dose Shenmai, respectively. The results showed that the patients who received high-dose Shenmai had significantly reduced white blood cells, CRP and procalcitonin, and increased peripheral blood CD4 + I CD8 + ratio [62]. In the treatment of sepsis, Shenmai significantly increased $\mathrm{CD} 3+, \mathrm{CD} 4+, \mathrm{NK}, \mathrm{IgM}$, and complement $\mathrm{C} 3$, and increased the value of $\mathrm{CD} 4+/ \mathrm{CD} 8+$, indicating that Shenmai can improve the immune function of patients [63]. These clinical functions of Shenmai might improve symptoms of critical patients with COVID-19 in terms of lung inflammation, virus infection, and drug-induced 
disease lung injury. However, there are no clinical studies in COVID-19. Currently, Shenmai is recommended for treating patients in the progressive stage of COVID-19 (critical case) in the NHC guidelines [6].

\section{Shengmai injection}

Shengmai is composed of red ginseng, ophiopogon japonicas, and schisandra chinensis [64]. Shengmai has often been used clinically to treat cardiovascular and cerebrovascular diseases. When Shengmai combined with conventional treatment in systemic inflammatory response syndrome, it can significantly increase the plasma prostacyclin (PGI2) and PGI2/TXA2, reduce the atrial natriuretic peptide (ANP) and endothelin (ET) level and improve breathing and vascular microcirculation. Shengmai plays a protective role in various organs [64]. One study observed the clinical effect of Shengmai on elderly patients with pulmonary infections and multiple organ failure. The results showed that adding Shengmai can significantly improve the cure rate of patients (treatment group: $83.9 \%$, oral Shengmai powder control group: $69.6 \%$, control group: $52.2 \%$ ), and shorten the improvement time and cure rate of multiple organ failure. Shengmai has the characteristics of rapid action and a remarkable curative effect [65]. Schisandra chinensis in Shengmai can increase the weight of spleen and lymph nodes in immunosuppressed mice caused by cyclophosphamide, increase the total volume of splenic white nucleus pulposus lymph node cortex, and enhance the body's immune function [66].

The above clinical functions of Shengmai might improve symptoms of critical patients with COVID-19 in terms of lung inflammation, virus infection, drug-induced disease lung injury. However, there are no reported clinical studies in COVID-19. Shengmai is currently recommended for treating patients in the progressive stage of COVID-19 (critical case) in the NHC guidelines [6].

\section{Discussion}

At present, the world is in a critical moment to defeat the COVID-19 pandemic. TCM's characteristics, past experiences in treating other viral infections, and several studies of TCM in treating COVID-19, TCM can contribute as an alternative measure. Based on this narrative review, TCM has many advantages in treating viral pneumonia and other emergent infectious diseases. Many TCMs have potent antiinflammatory and immunomodulatory effects. Treatment based on syndrome differentiation is one of the significant features in TCM [67]. TCM compounds contain a variety of active ingredients with proven multi-target effects, making it difficult for viruses to develop drug resistance. Through systematic analysis of previous clinical studies on the treatment of infectious pneumonia by TCM, it has been confirmed that TCM has sound therapeutic effects [68]. Because the etiology and pathogenesis are highly similar between COVID-19 and infectious pneumonia, clinicians can use TCMs to treat COVID-19 by referring to the rules of infectious pneumonia [69].

Among the oral and injectable TCMs we reviewed, Jinhua Qinggan and Lianhua Qingwen are available over-thecounter. It is essential to educate patients if the symptoms are not relieved or disappeared; they should seek medical care. At present, Xuebijing has been approved by the CFDA with a new indication of "treating for the new coronavirus pneumonia with severe and critical systemic inflammatory response syndrome or/and multiple organ failure". Lianhua Qingwen was approved to indicate "treating for fever, cough, and fatigue caused by the light and ordinary types of the new coronavirus pneumonia" [70].

Due to TCM's unique theoretical system, pharmacists as drug information expert, are well positioned to provide individualized pharmaceutical care based on the syndrome differentiation of the COVID-19 patient, especially for patients on the combined western medications and TCM treatments. Pharmacists play an active role in the review of TCM prescriptions, prepare TCM decoctions, TCM monitoring, patient education, science popularization, and clinical research [71].

This narrative review retrieved and reviewed relevant studies and documents to provide an objective analysis of the COVID-19 treatment utilizing TCMs. The review has the following limitation: the studies included were of varying types (e.g., case observation, clinical trials, blinded versus non-blinded). It was challenging to set general inclusion and exclusion criteria for which studies were included. Although the efficacy of some TCM remedies for COVID-19 is being tested, some trials have not been rigorously designed. Currently, fifteen trials listed in China's registry expect to enroll more than 2,000 people in studies to rigorously investigate the efficacy and safety of a variety of TCMs in COVID-19, including the most extensive trial assessing shuanghuanglian with 400 participants [72].

\section{Conclusion}

Under the guidance of TCM theory and the characteristics of TCMs, TCMs have been widely used in the treatment of COVID-19 in China. Both limited clinical studies and real-world experiences have indicated the role of TCMs in COVID-19 treatment.

Acknowledgements We like to thank all the authors whose articles are referenced in our study. 
Funding None.

Conflicts of interest The authors declare that they have no conflicts of interest.

\section{References}

1. Hick JL, Biddinger PD. Novel coronavirus and old lessonspreparing the health system for the pandemic. N Engl J Med. 2020;382(20):e55.

2. World Health Organize (WHO). WHO coronavirus disease (COVID-19) Dashboard [Internet]. Geneva: WHO (2020), August 7. https://www.who.int/docs/default-source/coronaviruse/situa tion-reports/20200505 covid-19-sitrep-106.pdf?sfvrsn=47090 f63_2.

3. The General Office of the National Health and Health Commission and the Office of the State Administration of Traditional Chinese Medicine. Notice on printing and distributing pneumonia diagnosis and treatment program for new coronavirus infection (Trial version 4) [Internet]. Beijing: China; 2020 January 28. www.gov. $\mathrm{cn} /$ zhengce/zhengceku/2020-01/28/content_5472673.htm. ISBN: National Health Office Medical Letter (2020) No. 77

4. The General Office of the National Health and Health Commission and the Office of the State Administration of Traditional Chinese Medicine. Notice on printing and distributing pneumonia diagnosis and treatment program for new coronavirus infection (trial version 5) [Internet]. Beijing: China; 2020 February 5. https:// www.gov.cn/zhuanti/2020-02/09/content_5476407.htm. ISBN: National Health Office Medical Letter (2020) No. 117

5. The General Office of the National Health and Health Commission and the Office of the State Administration of Traditional Chinese Medicine. Notice on printing and distributing pneumonia diagnosis and treatment program for new coronavirus infection (trial version 6) [Internet]. Beijing: China; 2020 February 19. https ://www.gov.cn/zhengce/zhengceku/2020-02/05/content_54747 91.htm. ISBN: National Health Office Medical Letter (2020) No. 145

6. The General Office of the National Health and Health Commission and the Office of the State Administration of Traditional Chinese Medicine. Notice on printing and distributing pneumonia diagnosis and treatment program for new coronavirus infection (trial version 7) [Internet]. Beijing: China; 2020 March 4. https://www.gov. cn/zhengce/zhengceku/2020-03/04/content_5486705.htm. ISBN: National Health Office Medical Letter (2020) No. 184

7. World Health Organization. Regional Office for the Western Pacific. WHO international standard terminologies on traditional medicine in the Western Pacific Region. Geneva: WHO; 2007. ISBN: 9789290612487

8. World Federation of Chinese Medicine Societies. International standard Chinese-English basic nomenclature of Chinese Medicine. Beijing: China; 2008.

9. Li D, Che Z. TCM syndrome type analysis of 58 cases of new crown pneumonia in Wuhan square cabin hospital, China. Trace Elem Health Res. 2020;5:40-1.

10. Ma J, Chen M, Wang YG. Summary of TCM syndrome and treatment of novel coronavirus pneumonia, China. Beijing. Tradit Chin Med J. 2020;39(02):95-101.

11. Zhang Q, Cao F, Wang Y, Xu X, Sun Y, Li J, et al. The efficacy and safety of Jinhua Qinggan granule (JHQG) in the treatment of coronavirus disease 2019 (COVID-19): a protocol for systematic review and meta analysis. Medicine (Baltim). 2020;99(24):e20531.
12. Qi JP, Qi XY, Wang XJ. The effect of different doses of Jinhua Qinggan granule on influenza and its effect on serum cytokine, China, Mod Med J. 2016;44(12):1664-9.

13. Duan C, Xia WG, Zheng CJ, Sun GB, Li ZL, Li QL, et al. Clinical observation of Jinhua Qinggan granule to treat COVID-19. J Tradit Chin Med. 2020;61:1-5.

14. Jia W, Wang C, Wang Y, Pan G, Jiang M, Li Z, et al. Qualitative and quantitative analysis of the major constituents in Chinese medical preparation Lianhua-Qingwen capsule by UPLC-DADQTOF-MS. Sci World J. 2015;2015:731765.

15. Liu CY, Li XQ, Cai SQ. Progress in pharmacology and clinical research of Lianhua Qingwen capsules, China. Pharm Clin Chin Mater Med. 2010;26(06):84-85+21.

16. Xia JW, Chen XJ, Zhang J, Zhou ZW. Experimental investigation of the effect of Lianhuaqingwen Capsule on the rat models of chronic obstructive pulmonary disease, China. Fudan Univ J Med Sci. 2008;03:441-4.

17. Ding Y, Zeng L, Li R, Chen Q, Zhou B, Chen Q, et al. The Chinese prescription lianhuaqingwen capsule exerts anti-influenza activity through the inhibition of viral propagation and impacts immune function. BMC Complement Altern Med. 2017;17(1):130.

18. Yao KT, Liu MY, Li X, Huang JH, Cai HB. Retrospective clinical analysis of traditional Chinese medicine Lianhua Qingwen in treating new coronavirus pneumonia, China. Chin J Exp Tradit Med Formul. 2020;26(11):8-12.

19. Cheng DZ, Wang WJ, Li Y, Wu XD, Zhou B, Song QY. Analysis of the efficacy of Chinese medicine Lianhua Qingwen in 51 patients with new coronavirus pneumonia: a multicenter retrospective study, China, Tianjin. Tradit Chin Med J. 2020;37(05):509-16.

20. Hu K, Guan WJ, Bi Y, Zhang W, Li L, Zhang B, et al. Efficacy and safety of Lianhua Qingwen capsules, a repurposed Chinese herb, in patients with coronavirus disease 2019: a multicenter, prospective, randomized controlled trial. Phytomedicine. 2020;16:153242.

21. Liu X, Zhang H, Xu J, Gong S, Han Y, Zhang T, et al. Identification of absorbed components and their metabolites in rat plasma after oral administration of Shufeng Jiedu capsule using ultra-performance liquid chromatography/quadrupole timeof-flight mass spectrometry. Rapid Commun Mass Spectrom. 2019;33(19):1494-501.

22. Ji S, Bai Q, Wu X, Zhang DW, Wang S, Shen JL, et al. Unique synergistic antiviral effects of Shufeng Jiedu capsule and oseltamivir in influenza A viral-induced acute exacerbation of chronic obstructive pulmonary disease. Biomed Pharmacother. 2020;121:109652.

23. Ji QJ, Shan RY, Deng SM, Tian YY, Chai W, Huang H, et al. Effect of Shu Feng Jiedu capsule on compliance of OSAHS patients before and after nCPAP treatment, China. World J Integ Tradit Western Med. 2019;14(05):692-4.

24. Ma L, Huang Y, Hou YB, Zhang DD, Zhang YP, Liu J, et al. Study on mechanism for immunoregulation of Shufeng Jiedu Capsule, Chiana. Drug Eval Res. 2019;42(09):1763-8.

25. Yang QW, Li Q, Zhang J, Xu Q, Yang X, Li ZY, et al.. Crystal structure and anti-inflammatory and anaphylactic effects of andrographlide sulphonate E in Xiyanping, a traditional Chinese medicine injection. J Pharm Pharmacol. 2019;71(2):251-9.

26. Liu YH, Feng F, Xie N. Advances in research on the anti-tumor, anti-pyretic and anti-inflammatory activity of andrographolide derivatives, China. Northwest Pharm J. 2013;28(1):95-8.

27. Shan M, Huang Y, Cui ZZ. Study on the effect of intravenous dripping of andrographolide in stabilizing body temperature of children with acute bronchial pneumonia, China. Chin Pediatr Integr Tradit Western Med. 2018;10(2):146-9.

28. Ding Y, Chen LZ, Wu WJ, Yang J, Yang ZF, Liu SW. Andrographolide inhibits influenza A virus-induced inflammation in a 
murine model through NF- $\mathrm{KB}$ and JAK-STAT signaling pathway. Microb Infect. 2017;19(12):605-15.

29. Cai N, Li YP, Zhou GJ, Xin C, Xie J, Zhou HH, et al. Theoretical basis and effect characteristics of andrographolide against COVID-19, China. Chin Tradit Herb Drug. 2020;51(05):1159-66.

30. Wang C, Shi QP, Ding F, Yu ML, Tang W, Cheng JQ. Reevaluation of the post-marketing safety of Xuebijing injection based on real-world and evidence-based evaluations. Biomed Pharmacother. 2019;109:1523-31.

31. Diao YF, Zhang SJ, Zhao WY, Sun HT, Zhang S, Sun L. Effect of Xuebijing Injection on expression of interleukin-6 and tumor necrosis factor- $\alpha$ in patients with severe pneumonia, China. Chin Tradit Herb Drug. 2017;48(06):1188-91.

32. Zhang CY, Zhang S, Wang W, Jiang XQ. Clinical observation of Xuebijing in the treatment of COVID-19, China. Chin J Hosp Pharm. 2020;40(09):964-7.

33. Sun K. The study of anti-inflammation mechanism of Reduning injection based on signaling pathways and discussion of disease therapy. Xian: Nothwest university; 2015.

34. Yan Y, Fan FJ, Zhao J, Bao JY, Feng LX. Clinical study on Reduning injection combined with biapenem in treatment of severe pneumonia. Drugs Clinic. 2019;34(12):3598-602.

35. Li HB, Yu Y, Wang ZZ, Xiao W, Yao XS. Research on antiviral constituents in Re-Du-Ning Injection (I),China. Chin Tradit Herb Drug. 2014;45(12):1682-8.

36. Zhang F, Sun L, Gao SH, Chen WS, Chai YF. LC-MS/MS analysis and pharmacokinetic study on five bioactive constituents of Tanreqing injection in rats. Chin J Nat Med. 2016;14(10):769-75.

37. Zhang B. Observation of therapeutic effect of tanreqing injection combined with ceftizoxime sodium for lower respiratory tract infection, China. Chin Tradit Herb Drug. 2009;40(6):947-8.

38. Zhu H, Chen M, Shi X, Shi C, Huang C. Material basis studies of anti-Influenza A active ingredients in Tanreqing injection. Biomed Chromatogr. 2018;32(2):e4097.

39. Wu Z, Chen C, Miao Y, Liu Y, Zhang Q, Li R, et al. Baicalin attenuates mycoplasma gallisepticum-induced inflammation via inhibition of the TLR2-NF- $\mathrm{BB}$ pathway in chicken and DF-1 Cells. Infect Drug Resist. 2019;20(12):3911-23.

40. Kong Y, Wu HW, Chen Y, Lai S, Yang ZM, Chen JS. Mechanism of Tanreqing Injection on treatment of coronavirus disease 2019 based on network pharmacology and molecular docking. China Chin Tradit Herb Drug. 2020;51(07):1785-94.

41. Pan W, Yang L, Feng W, Lin L, Li C, Liu W, et al. Determination of five sesquiterpenoids in Xingnaojing injection by quantitative analysis of multiple components with a single marker. J Sep Sci. 2015;38(19):3313-23.

42. Zhang AX. Application progress of Xingnaojing injection in clinical emergency, China. Mod J Integr Tradit Chin Western Med. 2013;22(17):1937-8.

43. Wu XL. Observation of anti-hyperthermia effect of Xingnaojing injection on adult upper respiratory tract infection, China. Shandong Med J. 2009;49(28):108-108.

44. Xu ZF, Wanf LJ, Yu ZM. Clinical Study on the intervention effect of Xingnaojing injection on acute infectious fever, China. Lingnan J Emerg Med. 2013;18(1):36-7.

45. Zhang JJ, Zhu ZY, Zhang J. The observation on therapeutic efficacy of antihyperthermal and revival effects of Xingnaojing Injection in treatment for severe craniocerebral injuries, China. Chin J Integ Tradit Western Med in Intensive Crit Care. 1999;6(1):5-7.

46. Wang J, Yang GT, Qiao LF. Effects of Xingnaojing injection on lipopolysaccharide-induces nuclear factor-kB activation and cytokine production in rat alveolar macrophages, China. Chin J Integr Tradit Western Med Int Crit Care. 2008;15(4):212-5.

47. Xie D, Hu J, Chen XH. Effect of Xingnaojing injection on the serum TNF- $\alpha$, CRP levels and immune function of elderly patients with mycoplasma pneumoniae pneumonia*, China. Prog Mod Biomed. 2016;16(16):3112-5.

48. Yin HY, Zhang R, Li BH. Effects of Xingnaojing injection on serum cytokines in patients with severe pneumonia in ICU, China. Chin J Clin Pharm Ther. 2007;12(12):1428-31.

49. Liu C, Hou Y, Wang X, Zhao Z, Liu Z, Zhai J, et al. Clinical assessment of Shenfu injection loading in the treatment of patients with exacerbation of chronic heart failure due to coronary heart disease: study protocol for a randomized controlled trial. Trials. 2015;21(16):222.

50. Zhang N, Liu JH, Qiu ZL, Ye YP, Zhang J, Lou TZ. Shenfu injection for improving cellular immunity and clinical outcome in patients with sepsis or septic shock. Am J Emerg Med. 2017;35(1):1-6.

51. Wang XJ, He CF, Cai YH, Sun DZ, Hu GY, Zhou J. Shen fu injection for patients with septic shock: protocol for an updated systematic review. Medicine (Baltimore). 2019;98(38):e17004.

52. Yu R. Clinical observation on infantile bacterial pneumonia treated by Shenfu injection, China. Chin Tradit Herb Drug. 2004;35(12):1397-8.

53. Hu Q, Yang WY, Long J. Effect of Shenfu injection on procalcitonin and immunoglobulin in elderly patients with chronic lung infection, China. J Emerg Tradit Chin Med. 2013;22(5):829-30.

54. Li N, Sheng KJ, Peng GX. Effect of Shenfu injection assisted with antibiotics on the subsided time of clinical symptoms, endothelial cell damage factor and pulmonary function in elderly patients with severe pneumonia, China. Prac Geriatr. 2019;33(8):764-7.

55. Li P. Hemodynamic effects of severe pneumonia by Shenfu injection, China. Guide Chin Med. 2012;10(33):52-3.

56. Liu X, Ai F, Li H, Xu Q, Mei LY, Miao JF, et al. Anti-Inflammatory effects of Shenfu injection against acute lung injury through inhibiting HMGB1-NF-kappaB pathway in a rat model of endotoxin shock. Evid Based Complement Altern Med. 2019;3(2019):9857683.

57. Zhang MY, Ji XF, Wang S, Li CS. Shen-fu injection attenuates postresuscitation lung injury in a porcine model of cardiac arrest. Resuscitation. 2012;83(9):1152-8.

58. Haijiang Z, Yongjiang W, Yiyu C. Analysis of 'SHENMAI' injection by HPLC/MS/MS. J Pharm Biomed Anal. 2003;31(1):175-83

59. Liu WY, Zhang JW, Yao XQ, Jiang C, He JC, Ni P, et al. Shenmai injection enhances the cytotoxicity of chemotherapeutic drugs against colorectal cancers via improving their subcellular distribution. Acta Pharmacol Sin. 2017;38(2):264-76.

60. Li L, Yang DL, Li JH, Niu L, Chen Y, Zhao X, et al. Investigation of cardiovascular protective effect of Shenmai injection by network pharmacology and pharmacological evaluation. BMC Complement Med Ther. 2020;20(1):112.

61. Fan XC, Tu S, Cao FB. Effect of Shenmai injection on inflammatory factors in patients with severe pneumonia, China. Hebei Med. 2018;24(10):1656-9.

62. Wang YF, Zhou Y, Li GF. Correlation between the dose of shenmai injection and the immune function and therapeutic effect of ventilator associated pneumonia, China. Chin J Mod Appl Pharm. 2019;36(24):3085-9.

63. Shen LE, Du QS, Zhao HL. Effect of Shenmai injection on immune function of patients with sepsis, China. Chin J Clin Ration Drug Use. 2014;7(1):67-8.

64. Guo Q, Zhang JQ, Shi R. Therapeutic effect of Shengmai injection combined with conventional Western medicine on systemic inflammatory response syndrome and its effect on vasoactive transmitter, China. Mod J Integr Tradit Chin Western Med. 2018;27(7):743-6.

65. Xu JN, Zhou CH, Huang SZ. Comparative observation on treatment of elderly pulmonary infection with multiple organ failure 
by adding Shengmai injection(Chinese). J Modern Clin Med. 1996;22(1):31-2.

66. Fei DL, Wang HJ, Su YG, Wang L. Effect of Schisandra sphenanthera polysaccharide on immune function of immunosuppressive mice induced by cyclophosphamide. China Feed Res. 2014;15:89-91.

67. Dong L, Li YQ, Yang SJ, Liu MN, Liu JL. A case study on syndrome differentiation and treatment of new coronavirus pneumonia. China Pharm Clin Chin Mater Med. 2020;36(02):69-71.

68. Ang L, Song E, Lee HW, Lee MS. herbal medicine for the treatment of coronavirus disease 2019 (COVID-19): a systematic review and meta-analysis of randomized controlled trials. J Clin Med. 2020;9(5):1583.

69. Zhao L, Zhao YF, Yuan ZY, Sun JB. Clinical application of Zhang Zhongjing's theory of "Treating Different Diseases with Same Method". China Tradit Chin Med Res. 2020;33(02):49-52.
70. Xinhuanet. Chinese medicine fights the "epidemic": from participant to main force [Internet]. Yu Y, editor. Shanghai: Xinhua News Agency. [cited 2020 July 28]. https://www.xinhuanet.com/ fortune/2020-02/19/c_1125595276.htm.

71. Meng L, Qiu F, Sun S. Providing pharmacy services at cabin hospitals at the coronavirus epicenter in China. Int J Clin Pharm. 2020;42(2):305-8.

72. Liu S, Luo P, Tang M, Hu Q, Polidoro JP, Sun S, et al. Providing pharmacy services during the coronavirus pandemic. Int J Clin Pharm. 2020;42(2):299-304.

Publisher's Note Springer Nature remains neutral with regard to jurisdictional claims in published maps and institutional affiliations. 